\title{
On single-row routing
}

\section{Andersen, Lars}

\section{Published in:}

IEEE Transactions on Circuits and Systems

Link to article, DOI:

10.1109/TCS.1980.1084751

Publication date:

1980

\section{Document Version}

Publisher's PDF, also known as Version of record

Link back to DTU Orbit

Citation (APA):

Andersen, L. (1980). On single-row routing. IEEE Transactions on Circuits and Systems, 27(12), 1262-1263. https://doi.org/10.1109/TCS.1980.1084751

\section{General rights}

Copyright and moral rights for the publications made accessible in the public portal are retained by the authors and/or other copyright owners and it is a condition of accessing publications that users recognise and abide by the legal requirements associated with these rights.

- Users may download and print one copy of any publication from the public portal for the purpose of private study or research.

- You may not further distribute the material or use it for any profit-making activity or commercial gain

- You may freely distribute the URL identifying the publication in the public portal

If you believe that this document breaches copyright please contact us providing details, and we will remove access to the work immediately and investigate your claim. 


\section{Letters to the Editor}

\author{
On Single-Row Routing \\ LARS ANDERSEN
}

\begin{abstract}
A new lower limit on the street congestion in single-row routing is presented.
\end{abstract}

\section{INTRODUCTION}

The single-row routing problem as presented in [1] and [2] includes several unsolved problems. This letter deals with only one of them.

Lower limits on the maximum street congestion are given both in [1] and [2]. In Section III it is shown that the limit given in [2] is often weaker than the one in [1], and a new lower limit is presented that may supersede these. This limit is based on the observation that in every interval covered by two or more nets one must be at the top and another at the bottom.

In this paper only nets connected to two and only two nodes will be considered. It is hoped that this limitation may be removed at a later time.

The use of the isomorphic mapping of a realization called the interval graphs was introduced in [2]. This and other terms and definitions from [2] are used in the following. However, for completeness most of the terms used later are defined in Section II.

\section{Definitions}

1) The terminals of a net are the nodes to which the net is connected.

2) A basic interval $b_{i}$ is the open interval of the reference line between the two successive nodes $i$ and $i+1$.

3) The density $d_{i}$ of a basic interval is the number of nodes passing over and/or under $b_{i}$.

Note: Nets placed on the reference line (only nets between two successive nodes) are not incalculated in $d_{i}$ because they do not influence the street congestion.

4) A net is said to cover a node if it passes over or under this node without being connected to it.

5) The range of a net is the set of nodes consisting of the nodes covered by the net and its terminals.

6) The cutnumber of a node $c_{i}$ is the number of nets covering node $i$.

7) The cutnumber of a net $q_{N}$ is the bigger of the cutnumbers of its terminals.

8) The length of a net $l_{N}$ is the number of nodes in its range.

9) The maximum street congestion $Q$ is the maximum number of nets that pass any basic interval on one side.

10) An outer node is a node that on one side is not covered by any net in a given realization.

11) An outer net in a basic interval $b_{i}$ is a net that on one side is not passed by any other net within $b_{i}$.

12) Let the net $N$ pass the basic interval $b_{i}$. Let the smallest cutnumber for the nodes in the range of $N$ to the left of $b_{i}$ be $c_{N i}^{\prime}$

Manuscript received October 15, 1979; revised February 19, 1980.

The author is with the Institute of Circuit Theory and Telecommunication, Technical University of Denmark, Lyngby, Denmark. and the smallest to the right of $b_{i}$ be $c_{N i}^{\prime \prime}$. To every such $b_{i}$ and $N$ is connected a condition number $y_{N i}$ defined as $y_{N i} \triangleq \max \left\{c_{N i}^{\prime}, c_{N i}^{\prime \prime}\right\}$.

13) For every basic interval $b_{i}$ with density $d_{i}>1$ we define a $Y_{i}$ as the smallest integer such that at least two nets have $y_{N i} \leqslant Y_{i}$.

\section{THEOREMS}

In [1] it was shown that

$$
Q \geqslant\left[d_{M} / 2\right]
$$

where $d_{M}=\max d_{i}$ and $[x]$ is the smallest natural number not smaller than $x^{i}$.

In [2] it was shown that

$$
Q \geqslant \max \left\{q_{m},\left[q_{M} / 2\right]\right\}
$$

where $q_{m}=\min _{N} q_{N}$ and $q_{M}=\max _{N} q_{N}$.

Let the net $N$ have $q_{N}=q_{M}$ and let node $i$ be a terminal of $N$ with $c_{i}=q_{M}$. The basic intervals $b_{i-1}$ and $b_{i}$ must have either

$$
d_{i}=q_{M} \text { and } \quad d_{i-1}=q_{M}
$$

or

$$
d_{i}=q_{M} \text { and } d_{i-1}=q_{M}+1
$$

or

$$
d_{i}=q_{M}+1 \text { and } d_{i-1}=q_{M} .
$$

(The first case only if net $N$ is on the reference line.) From this it is seen that

$$
d_{M} \geqslant q_{M}
$$

and

$$
\left[d_{M} / 2\right] \geqslant\left[q_{M} / 2\right] \text {. }
$$

Therefore, (2) should be replaced by

$$
Q \geqslant \max \left\{q_{m},\left[d_{M} / 2\right]\right\} \text {. }
$$

Theorem 1.

For an outer node $c_{i} \leqslant Q$.

Proof: On one side this node is not covered by any nets. Therefore all the $c_{i}$ covering nets must be in either the upper or the lower street.

Corollary 1: A net that is terminated in two outer nodes must have $q_{N} \leqslant Q$.

Theorem 2.

If the net $N$ is an outer net in the entire hasic interval $b_{i}$ then it has a condition number not greater than $Q$ in this interval:

$$
y_{N i} \leqslant Q \text {. }
$$

Proof: It is sufficient to show that the range of $N$ contains at least one node at each side of $b_{i}$ with cutnumber $c_{i} \leqslant Q$.

Only the nodes to one side (left) of $b_{i}$ are considered as the other side (right) can be treated in the same way.

Let $N$ be an upward outer net in $b_{i}$. If the left terminal $\left(T_{L}\right)$ of net $N$ is an outer node then $c_{i}$ of this node is $\leqslant Q$ according to Theorem 1.

If $T_{L}$ is not an outer node then it is upward covered by at least one net (see Fig. 1). Such upward covering nets must have the right terminals $\left(T^{\prime}\right)$ between $T_{L}$ and $b_{i}$ where $N$ is the upward 


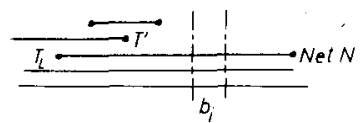

Fig. 1. Interval graph illustrating Theorem 2 .

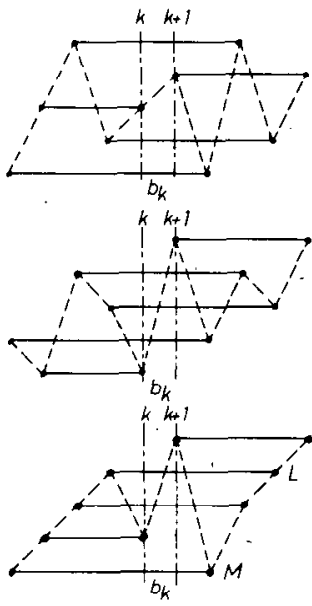

Fig. 2. Interval graph illustrating Theorem 3. (a) Two outer nets. (b) Two outer nodes. (c) One outer net and one outer node.

outer net. If $T^{\prime}$ is an outer node it has a $c_{i} \leqslant Q$. If $T^{\prime}$ is upward covered then the reasoning can be repeated. As there is a finite number of nodes between $T_{L}$ and $b_{i}$ it follows that this repetition will end with some node in the range of $N$ that is an outer node and, therefore, with $c_{i} \leqslant Q$.

Theorem 3.

$$
Y_{M} \leqslant Q
$$

where $Y_{M}=\max Y_{i}$ for all $i=1,2, \cdots, n-1$ where $d_{i}>1$

Proof: For each basic interval $b_{k}$ the set of surrounding nodes $(k, k+1)$ must fall in one of the following categories (see Fig. 2):

a) No outer nodes,

b) Two outer nodes, or

c) One outer node.

In each case it will be shown that there are two nets with $y_{N k} \leqslant Q$ and, therefore, $Y_{k} \leqslant Q$.

Case a: As none of the nodes $k$ and $k+1$ are outer nodes, they must be covered at both sides by two outer nets. Theorem 2 tells that $y_{N k} \leqslant Q$ for both nets.

Case $b$ : As both the nodes $k$ and $k+1$ are outer nodes Theorem 1 tells that $c_{k} \leqslant Q$ and $c_{k+1} \leqslant Q$. All nets passing $b_{k}$ will, therefore, have $y_{N k} \leqslant Q$.

Case $c:$ As only one node is an outer node (say node $k+1$ ), the other node $(k)$ must be covered on both sides (both upward and downward). One of these nets is an outer net in $b_{k}$ while some other is crossing the reference line inside $b_{k}$.

The outer net $(M)$ has $y_{M k} \leqslant Q$ (Theorem 2). One of the nets crossing the reference line within $b_{k}$ is an outer net (say net $L$ ) in the neighboring basic interval $b_{k-1}$. Therefore, $y_{L k-1} \leqslant Q$ which means that a node in its range to the left of $b_{k-1}$ (and thereby to the left of $b_{k}$ ) has a cutnumber $c_{i} \leqslant Q$. The node $k+1$ is in the range of net $L$ to the right of $b_{k}$ and its $c_{k+1} \leqslant Q$ because it is an outer node. In other words, also $y_{L k} \leqslant Q$.

The requirement $q_{m} \leqslant Q$ in (2) and (3) is the result of Corollary

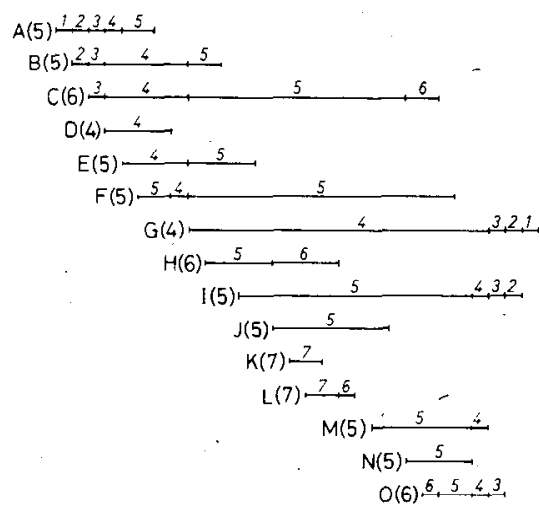

$\sigma_{i} \quad 12345654565656787656567654371$

$c_{j} 012345544555556776555566543210$

$2,3,4$

$$
d_{M}=8 \quad V_{M}=5
$$

Fig. 3. $d_{i}, c_{i}, q_{N}, y_{N i}, Y_{i}, d_{M}$, and $Y_{M}$ for Example 2 of [2].

Theorem 3 is the result of observations on all basic intervals and their outer nets.

Although it is not proven that $q_{m} \leqslant Y_{M}$ it is suggested that (2) and (3) should be replaced by

$$
Q \geqslant \max \left\{Y_{M},\left[d_{M} / 2\right]\right\} \text {. }
$$

\section{Discussion}

Fig. 3 shows the original interval graph of Example 2 from [2] with the $y_{N i}$ 's written on each net and with the $d_{i}, c_{i}$, and $Y_{i}$ indicated below.

From this it is seen that $Y_{M}=5$ and that it will be futile to search for realizations with $Q=4$.

\section{REFERENCES}

[1] B. S. Ting, E. S. Kuh, and I. Shirakawa, "The multilayor routing problem: Algorithms and necessary and sufficient conditions for the single-row, single-layer case," IEEE Trans. Circuits Syst., vol. CAS-23, pp. 768-778, Dec. 1976.

[2] E. S. Kuh, T. Kashiwabara, and T. Fujisawa, "On optium single-row routing," IEEE Trans. Circuits Syst., vol. CAS-26, pp. 361-368, June 1979.

\section{On the Periodic Steady-State Problem by the Newton Method}

DAVID A. ZEIN, SENIOR MEMBER, IEEE

Abstract-The methods by which linear sensitivity circuits have been used to evaluate the sensitivity matrix, $\partial x(T) / \partial x(0)$, can be improved. The sensitivity matrix is used in the computation of the Jacobian of $f(x(0))=x(0)-x(T)=0,(T$ being the period) in a periodic steady-state problem. It is shown here that the sensitivity matrix can be obtained from the solution of a linear homogeneous matrix equation which is simply derived by differentiating the state equations or a mixture of algebraicdifferential equations arising from any formulation. This simplification makes the method easier and more practical to implement in a general purpose CAD program. Examples are given.

Manuscript received May 22, 1979; revised January 28, 1980

The author is with the IBM Data System Division, Hopewell Junction, NY 12533. 